\title{
Journal of Clinical Monitoring and Computing 2018-2019 end of year summary: respiration
}

\author{
D. S. Karbing ${ }^{1} \cdot$ G. Perchiazzi ${ }^{2}$ S. E. Rees ${ }^{1} \cdot$ M. B. Jaffe ${ }^{3}$
}

Received: 21 January 2020 / Accepted: 21 January 2020 / Published online: 24 January 2020

(c) Springer Nature B.V. 2020

\begin{abstract}
This paper reviews 28 papers or commentaries published in Journal of Clinical Monitoring and Computing in 2018 and 2019, within the field of respiration. Papers were published covering endotracheal tube cuff pressure monitoring, ventilation and respiratory rate monitoring, lung mechanics monitoring, gas exchange monitoring, $\mathrm{CO}_{2}$ monitoring, lung imaging, and technologies and strategies for ventilation management.
\end{abstract}

Keywords Review $\cdot$ Respiration $\cdot$ Monitoring $\cdot$ Endotracheal tube cuff pressure $\cdot$ Ventilation $\cdot$ Lung mechanics $\cdot \mathrm{O}_{2}$ and $\mathrm{CO}_{2}$ exchange $\cdot$ Lung imaging

\section{Introduction}

In the period 2018-2019, the Journal of Clinical Monitoring and Computing published 28 papers or commentaries in the field of respiration, these papers encompassing endotracheal tube cuff pressure monitoring, ventilation and respiratory rate monitoring, lung mechanics monitoring, gas exchange monitoring, $\mathrm{CO}_{2}$ monitoring, lung imaging, and technologies and strategies for ventilation management. In comparison to 2017 [1], the journal again published several papers on ventilation, gas exchange and $\mathrm{CO}_{2}$ monitoring, but with an increased focus on endotracheal tube cuff pressure management and lung imaging.

D. S. Karbing

dank@hst.aau.dk

1 Respiratory and Critical Care Group (Rcare), Department of Health Science and Technology, Aalborg University, Aalborg, Denmark

2 Department of Surgical Sciences, The Hedenstierna Laboratory, Uppsala University, Uppsala, Sweden

3 Cardiorespiratory Consulting, LLC, Cheshire, CT, USA

\section{Endotracheal tube cuff pressure monitoring}

Tracheal intubation to establish mechanical ventilation via an endotracheal tube (ETT) is a common practice both in the operating room and the intensive care unit [2]. The endotracheal tube has a cuff which must be inflated to seal the airway and prevent leaks and secretion aspiration. It is important to maintain the pressure of the ETT cuff within a range of 15-25 mmHg (or 20-30 $\mathrm{cm} \mathrm{H}_{2} \mathrm{O}$ ) [2, 3]. Too low ETT cuff pressure increases risk of ventilator associated pneumonia whereas too high pressure may lead to damaging the tracheal tissue [2, 3]. In 2018-2019, the journal published three papers on ETT cuff pressure monitoring covering reliability of ETT cuff pressure assessment by palpation using a regular and an alternate pilot balloon, the effect of a training seminar on the palpation technique and reliability of ETT cuff pressure gauges.

Pisano et al. [4] studied the reliability of detecting ETT cuff overinflation by the finger palpation technique, where a pilot balloon connected to the cuff is used to assess cuff inflation state. In a simulated trachea test setup, 41 experienced and 21 resident anesthetists were asked to classify cuff pressure as too much, adequate or too low in three scenarios; using a normal pilot balloon at significantly overinflated state $\left(88 \mathrm{mmHg}, 120 \mathrm{~cm} \mathrm{H}_{2} \mathrm{O}\right)$, using a larger pilot balloon at same overinflation, and the large balloon at less significant overinflation ( $40 \mathrm{mmHg}, 54 \mathrm{~cm} \mathrm{H}_{2} \mathrm{O}$ ). Only $35 \%$ of participants detected considerable overinflation 
using a normal balloon versus $87 \%$ using the larger balloon with answers being significantly different $(\mathrm{P}<0.0001)$ and only answers using the larger balloon being significantly different from answers at random, regardless of seniority $(\mathrm{P} \leq 0.0001)$. An overall of $32 \%$ were able to detect too much inflation using a larger balloon at the less overinflated state, with less experienced anesthetist performing worse than random $(\mathrm{P}=0.0043)$. The authors concluded that whilst the results show a greater reliability of the palpation technique using a larger balloon, the larger balloon could not increase reliability of the technique to a clinically satisfactory level.

In their prospective observational study, Özcan et al. [5] investigated the effect of a training seminar on ETT cuff pressure management by finger palpation in elective surgery patients. The authors compared cuff pressure measurements in 100 patients 1 week prior to with measurements in 100 patients one week following a training seminar. Assistant anesthesiologists were in charge of ETT cuff inflation in both groups, and all attended the same 30 min training seminar. They were blinded as they were unaware of the study being performed. Following training measured ETT cuff pressures were significantly lower at median (IQR) $32(15) \mathrm{cm} \mathrm{H}_{2} \mathrm{O}$ compared to 48 (30) $\mathrm{cm} \mathrm{H}_{2} \mathrm{O}$ measured prior to the seminar $(\mathrm{P}<0.001)$. The two patient groups did not differ significantly in terms of age, gender, or tube diameters $(\mathrm{P}>0.05)$. ETT cuff pressure did not differ significantly between assistant anesthesiologists of different seniority. The authors argued that frequent training activities and reminders will ensure optimal cuff pressure, but also that the observed high ETT cuff pressures in their study suggest that ETT cuff pressure should be monitored, preferably with objective measurements. It will be interesting to see if future studies can demonstrate a training effect beyond the 1 week studied by Özcan et al.

ETT cuff pressure monitoring by pressure gauges are preferred to finger palpation for ETT cuff monitoring [3]. Raft et al. [6] studied reliability of 27 pressure gauges from 3 manufacturers (Mallinckrodt, Covidien and Portex) that were used daily in operating rooms at their hospital. Pressure gauges were tested against a pressure gauge calibrator, each device evaluated at seven pressure levels between 0 and $50 \mathrm{~cm} \mathrm{H}_{2} \mathrm{O}$. Only $30 \%$ of gauges fulfilled homologation criteria consisting of passing a leak test and a pressure gauge and calibrator difference $<1.3 \mathrm{~cm} \mathrm{H}_{2} \mathrm{O}$ in cuff pressures of $20-30 \mathrm{~cm} \mathrm{H}_{2} \mathrm{O}$. The authors concluded that there was a problem with reliability and follow-up in the pressure gauges they had tested at their hospital leading them to suggest that regular and mandatory follow-up of pressure gauges should be performed and that future studies should investigate how cuff pressure gauges are monitored. Whilst intermittent ETT pressure monitoring with pressure gauges may be preferred to finger palpation, options for automatic continuous regulation also exist and some data have been reported on the effectiveness of such solutions [for review see 3].

\section{Ventilation and respiratory rate monitoring}

Four papers were published in the journal in the past 2 years relating to ventilation and respiratory rate monitoring. They cover the performance evaluation of an ambulatory device for detection of obstructive sleep apnea (OSA), the assessment of a thermal imaging system to detect apnea in patients undergoing spinal anesthesia, the apnea detection performance in the post anesthesia care unit (PACU) of an microphone based acoustic method, and a study of the use of rate of low minute ventilation events as measured with a bioimpedance monitor to assess respiratory depression in the PACU.

Faßbender et al. [7] evaluated the accuracy of OSA measures from a wrist mounted device (SomnoCHeck micro, Weinmann, Hamburg Germany) against a laboratory polygraphy device (Somno Lab 2) from the same company in patients with known or suspected OSA who have been scheduled for surgery. The wrist mounted device uses a nasal cannula to detect both snoring and nasal air flow and a finger sensor for photoplethysmography (PPG). The primary measure used for comparison of the devices was a computed Apnea/Hypopnea Index (AHI) (respiratory events per hour) based on standard flow/oxygen saturation based apnea and hypopnea criteria. Central and obstructive events were distinguished using a thoracic strap in the lab system and in the wrist system by analysis of the PPG fluctuations related to changes in intrathoracic pressure [8]. For the 48 patients studied, a good correlation $\left(r^{2}=0.723\right)$ and mean difference of 3.1 between the AHI from the two systems was found. The system described in this paper is now one of many nonlaboratory diagnostic systems for apnea on the market [9] with the majority intended for home based sleep testing (i.e. Type 3 and 4 sleep tests [10]).

Kim et al. [11] assessed the ability of thermal images of the patient's face, specifically the region around the nostrils, as recorded by an infra-red camera (IRT) to detect apnea in 20 patients undergoing spinal anesthesia during endoscopic urological surgery. Thermal images of fluctuations in the region around the nostrils during the respiratory cycle were measured at a 10 frames/s. Respiratory waveforms were extracted from these regions near the nostrils caused by respiration. Frame to frame feature tracking using the nose as an anchor point was required to align and correct for movements of the head. Respiratory rate (RR) determined via the IRT as compared to RR from a bioimpedance based respiratory volume monitor (RVM) showed a good correlation 
$\left(r^{2}=0.75\right)$. Additionally, the speed relative to the RVM that IRT can detect the changes in respiration was assessed with the authors reporting that IRT detected changes $40 \mathrm{~s}$ earlier than RVM, although the authors suggested that this comparison could be problematic and related to the averaging window used for RR. Technologies suited for non-contact respiratory monitoring such as infrared imaging and others such as ultrawide band radar are increasingly being explored for use in clinical and nonclinical environments (e.g. home and auto) where patient comfort and mobility are paramount $[12,13]$.

Liu et al. [14] assessed the apnea detection performance of an acoustic method in patients with frequent apneic events in the PACU against events determined with a processed nasal pressure signal, shown to be proportional to square of flow. Signals from an off the shelf microphone encased in plastic (with $-3 \mathrm{~dB}$ frequency response of $100-5000 \mathrm{~Hz}$ ) was low pass filtered to $150-800 \mathrm{~Hz}$ to minimize the impact of heart sounds, muscle interference and high frequency noise and the log of tracheal sound variance was computed to detect apneic events. The study included 120 patients with 34 patients experiencing 236 events lasting a total of $122.2 \mathrm{~min}$. The authors' note that the algorithm's performance of $92 \%$ sensitivity and $98 \%$ specificity exceeds that of a piezo-electric film based acoustic method reported in the literature. Using tracheal sounds for apnea detection is an area of increased research and commercial interest, particularly with respect to the detection of sleep apnea [15].

Cavalcante et al. [16] performed a prospective observational study of measures of respiratory depression in the PACU. Patients who received anesthesia for intraperitoneal surgery were studied and the rate of low minute ventilation events (LMVe) during recovery determined. LMVe was measured using a non-invasive bioimpedance respiratory volume monitor (ExSpiron $1 \mathrm{Xi}$, Respiratory Motion Inc., Waltham, MA) which was calibrated using the current minute ventilation (MV) value from the anesthesia machine. An LMVe was considered to occur if MV is less than $40 \%$ of the body surface area predicted minute ventilation and sustained for at least $2 \mathrm{~min}$. In the 107 patients studied 36\% had at least one LMVe with the LMVe's occurring with a median of 6 episodes per patient and median duration of $4.2 \mathrm{~min}$. The authors also examined the utility of using low RR, defined as $\mathrm{RR}<8$ breaths/min, to detect respiratory depression and noted in these patients it was not useful for detecting respiratory depression having missed $88.2 \%$ of all low $\mathrm{MV}(<40 \%$ of predicted MV) events. Patients receiving opioids during recovery were assessed for opioid induced respiratory depression (OIRD), defined as an LMVe occurring within 30 min of the first PACU administration of the opioid. OIRD was reported in 27 of the 45 patients receiving opioids. The authors highlighted the association of LMVe's and opioids in the PACU in patients with pre-existing mild respiratory depression which was defined as $\mathrm{MV}<70 \%$ predicted and found to be the best predictor of OIRD of the measures assessed. This study lends further support to the notion that respiratory rate alone may not be adequate to assess and identify respiratory depression in patients in the PACU [17].

\section{Lung mechanics monitoring}

Accurate assessment of lung mechanics is complicated by the difficult access to the lungs and the many interdependent components of the respiratory system. Monitoring and diagnostics therefore often require invasive measurements and/ or respiratory maneuvers to be performed. In 2018-2019, the journal published three papers on lung mechanics monitoring covering measurement of lung mechanics for lung function assessment, in mechanically ventilated severely ill patients during, and in mechanically ventilated patients with spontaneous breathing efforts. These were all concerned with making lung mechanics measurement more accessible.

The forced oscillation technique (FOT) is a non-invasive method for assessing lung mechanics. It consists of superimposing small-amplitude pressure oscillations on the normal breathing and has the benefit of not requiring any respiratory manoeuvres as opposed to conventional spirometry tests [18]. Zimmermann et al. [19] compared airway resistance and reactance at measurements at $5 \mathrm{~Hz}$ by 4 different FOT devices (a custom-built device, Resmon Pro Diary (Restech srl, Italy), tremoFlo C-100 (Thorasys Medical Systems, Canada), and Jaeger Masterscope CT IOS (CareFusion, Hoechberg, Germany)). In vivo testing in 12 healthy subjects showed no difference in resistance between devices, with $<10 \%$ deviation from predicted normal values, except for the Jaeger Masterscope CT IOS. The custom-built device and Resmon Pro Diary gave similar values for reactance, as did the tremoFlo C-100 and Jaeger Masterscope CT IOS with other pairwise comparisons being different $(\mathrm{P}<0.05)$. An in vitro test in two test standards showed no difference between devices $(\mathrm{P}>0.05)$ with deviations from theoretical values $<2 \%$ for resistance and $<5 \%$ for reactance. The authors concluded that the observed in vivo differences between device measurements in their study highlight the need for validation procedures taking into account breathing pattern or volume changes which is neglected by current methods.

Spontaneous breathing efforts during support mode mechanical ventilation make assessment of respiratory system compliance (Crs) difficult due to the unknown pressure generated by respiratory muscles. Becher et al. [20] described a method for calculating Crs during pressure support mode from volume changes induced by repeated small changes in pressure. They prospectively evaluated the method in 20 patients. Measurements using the new method 
in pressure support ventilation were compared to reference measurements obtained in the same patients during volume control ventilation without spontaneous efforts. The authors found good correlation between the new method and reference measurements but with relatively high bias of $-7 \mathrm{ml} /$ $\mathrm{cm} \mathrm{H}_{2} \mathrm{O}$ and broad 95\% limits of agreement of -16.7 to $+2.7 \mathrm{ml} / \mathrm{cm} \mathrm{H}_{2} \mathrm{O}$. Analysis of the last $20 \%$ of the reference measurements yielded smaller bias but broader limits of agreement. The authors concluded that the new method may, despite limited accuracy, be useful for non-invasive Crs monitoring during pressure support ventilation. As recognised by the authors, routine application would require automation and ventilator integration of the 10-15 changes in pressure support performed manually in their study.

Stress index (SI), which is calculated from the shape of the airway pressure-time curve in volume control ventilation, describes the average changes in Crs over an inspiratory time period, and was originally proposed by Ranieri et al. as a bedside parameter to minimize for lung protective mechanical ventilation [21]. SI measurement requires device and software which is not readily available for all ventilators. Wongsurakiat et al. [22] therefore investigated the feasibility of estimating SI by visual inspection of the airway pressure-time curve. Visual inspection by two physicians were compared with measurements by dedicated software in 30 patients with acute respiratory distress syndrome (ARDS) at peak inspiratory flows of 60 and $40 \mathrm{l} / \mathrm{min}$. Overall sensitivity of visual inspection to identify breathing as either tidal recruitment $(\mathrm{SI}<0.9)$, noninjurious ventilation $(0.9 \leq \mathrm{SI} \leq 1.5)$ or tidal overdistension $(\mathrm{SI}>1.05)$ was higher for peak inspiratory flow of $40 \mathrm{vs} 60 \mathrm{l} / \mathrm{min}(75 \%$ vs. 50\%; $\mathrm{P}=0.005$ ). Experience of physicians did not affect sensitivity significantly. The authors concluded that SI estimation by visual inspection was moderately accurate and could be used in conjunction with the more widely available plateau pressure for monitoring of lung mechanics in ARDS patients.

\section{Gas exchange monitoring}

In the past 2 years, the journal published three papers on gas exchange monitoring, covering three steps of the transport pathway of respiratory gases: the mixing of alveolar gases as monitored by end-expired oxygen fraction, the contributing factors to gas exchange between alveolar air and lungcapillary blood and the $\mathrm{O}_{2}, \mathrm{CO}_{2}$ and acid-base status of arterial blood. In common for all papers was the application of mathematical physiological models.

Measurement of end-tidal $\mathrm{O}_{2}$ fraction $\left(\mathrm{F}_{\mathrm{ET}} \mathrm{O}_{2}\right)$, can be used to evaluate prexoygenation prior to induction of anesthesia in surgery patients [23]. Burk et al. [24] described a new method for estimating nasal $\mathrm{F}_{\mathrm{ET}} \mathrm{O}_{2}$, which is otherwise difficult during administration of oxygen. The method comprises of switching off nasal $\mathrm{O}_{2}$ flow temporarily to analyse $\mathrm{F}_{\mathrm{ET}} \mathrm{O}_{2}$ from the first three washout breaths to extrapolate backwards and estimate the enhanced $\mathrm{F}_{\mathrm{ET}} \mathrm{O}_{2}$ during administration of nasal $\mathrm{O}_{2}$ flow. Eighteen breath washout conditions were evaluated using a bench setup consisting of an upper airway replica and a test lung connected to a ventilator. Bias and $95 \%$ limits of agreement between calculated and observed $\mathrm{F}_{\mathrm{ET}} \mathrm{O}_{2}$ were -0.016 and -0.048 to 0.016 , respectively. Extrapolations from washout breath 1 were evaluated in 30 healthy volunteers, yielding bias and limits of agreement for calculated versus observed $\mathrm{F}_{\mathrm{ET}} \mathrm{O}_{2}$ of 0.003 and -0.017 to 0.023 for breaths 2 and 3 , and -0.005 and -0.042 to 0.033 for breath 4 . Then the authors used the method for comparison of two different techniques for nasal oxygen administration (pulsed and continuous flow). This comparison, which was performed in the same healthy volunteers, showed that pulsed flow could achieve the same $\mathrm{F}_{\mathrm{ET}} \mathrm{O}_{2}$ as the continuous flow technique using on average only a third of the oxygen flow.

The multiple inert gas elimination technique (MIGET) is generally considered the reference technique for estimating shunt, ventilation/perfusion mismatch and dead space lung gas exchange parameters [25]. However, the technique is complex and time-consuming. The MIGET-MMIMS method shortens the time requirement to approximately 15 min by replacing gas chromatography with micropore membrane inlet mass spectrometry (MMIMS) and the technique has been shown to correlate well with MIGET [26]. Gerber et al. [27] compared MIGET-MMIMS estimated shunt and dead space values with the more common Riley shunt and Bohr dead space. Thirty pigs were subjected to lavage or pulmonary embolism induced lung injury followed by four hours of measurements, yielding data from 15 and 10 pigs, respectively. MIGET-MMIMS resulted on average in lower shunt with a bias and $95 \%$ limits of agreement of -0.05 and -0.15 to 0.04 and $r^{2}$ of 0.788 . MIGET-MMIMS resulted on average in lower dead space with a bias and limits of agreement of -0.09 and -0.24 to 0.05 and $r^{2}$ of 0.56 . MIGET-MMIMS shunt increased significantly after injury by lavage only, whereas MIGET-MMIMS dead space increased significantly in both groups $(\mathrm{P}<0.05)$.

Lumholdt et al. [28] (corrected in [29]) evaluated a commercially available method for model-based calculation of arterial blood gas values from a venous blood gas sample and pulse oximetry (v-TAC, Obimedical, Denmark [30]) sparing patients from the relatively more painful arterial puncture. In 20 emergency department patients, values calculated using the v-TAC method were compared with values from arterial blood gas samples. Venous blood samples were analysed within 5 min after sampling, with the sample tilted and analysed after $7 \mathrm{~min}$, and with the sample steady and analysed after $15 \mathrm{~min}$. Acceptable thresholds for deviance from arterial blood gas values were defined 
as $\pm 0.05 \mathrm{pH}$ units, $\pm 0.88 \mathrm{kPa} \mathrm{CO}$ partial pressure $\left(\mathrm{PaCO}_{2}\right)$ and $\pm 0.88 \mathrm{kPa} \mathrm{O}$ partial pressure $\left(\mathrm{PaO}_{2}\right)$. Tolerability interval ratios, defined as $95 \%$ limits of agreement as proportion of acceptable interval, were for the three methods 0.64-0.77 for $\mathrm{pH}, 0.48-0.63$ for $\mathrm{PaCO}_{2}$ and 2.94-3.84 for $\mathrm{PaO}_{2}$. As such, agreement was found for $\mathrm{pH}$ and $\mathrm{PaCO}_{2}$, but not $\mathrm{PaO}_{2}$. Concordance correlation coefficients were $0.744-0.942$ for $\mathrm{pH}, 0.639-0.935$ for $\mathrm{PaCO}_{2}$ and $0.652-0.720$ for $\mathrm{PaO}_{2}$. The authors concluded that calculated values were valid for $\mathrm{pH}$ and $\mathrm{PaCO}_{2}$ but not $\mathrm{PaO}_{2}$, and that larger clinical studies were required to address use of the method in different patient subpopulations.

\section{$6 \mathrm{CO}_{2}$ monitoring}

Five papers were published in the journal in the past 2 years relating to $\mathrm{CO}_{2}$ monitoring. They include a performance evaluation with respect to nasal and oral $\mathrm{CO}_{2}$ monitoring of patients using a new mandibular advancement bite block with a winged extension, a pilot study in patients with suspected obstructive sleep apnea of an airway obstruction prediction algorithm (RESDA) using end-tidal $\mathrm{CO}_{2}$ and mandibular movement signals, a collection of surgical case summaries that illustrate the utility of changes in the shape of the capnogram in rapidly assessing pulmonary perfusion in patients undergoing a lung lobectomy, and performance studies from two different groups on their variants of the differential Fick method for pulmonary blood flow termed capnodynamic and capnotracking methods.

Teng et al. [31] compared the impact on the measured capnogram of a standard reusable bite block and mandibular advancement (MA) bite block with a winged extension in patients undergoing upper gastrointestinal endoscopy. The standard bite block required the use and placement of separate catheters for nasal and oral gas sampling and nasal oxygen delivery while the mandibular bite block provided a connection port for oxygen that distributed it to both the oral and nasal cavities and a gas sampling port. The end-tidal $\mathrm{CO}_{2}$ values from 119 patients divided between the two types of bite blocks ( $\mathrm{n}=30$ standard, $\mathrm{n}=89$ MA bite block) were compared. Measured end-tidal $\mathrm{CO}_{2}$ values were determined for each patient in three states-awake, sedated before the procedure and sedated during the procedure. With the standard bite block, the oral and nasal values on average varied widely, while the MA bite block showed relatively consistent values on average between the oral and nasal readings and three states. The authors observed that most patients are mouth breathers in sedated upper GI endoscopy and that although oral supplementary oxygen dilutes the gas and reduces the measured end-tidal $\mathrm{CO}_{2}$ value it remains useful. This bite block design as well as several of the commercially available bite blocks with integrated gas sampling ports (e.g.
Smart Sureline Guardian Bite Block, Covidien;DualGuard, Flexicare; Safety-Guard, Vygon; TwinGuard, Trawax) may allow for more accurate capnographic recordings and should be considered for use when monitoring $\mathrm{CO}_{2}$ during upper endoscopic procedures [32].

Ponthieu et al. [33] reported on a pilot study on the performance of an airway obstruction prediction algorithm (RESDA) on 21 adult patients with suspected obstructive sleep apnea who were scheduled for endoscopy. The RESDA algorithm inputs include end-tidal $\mathrm{CO}_{2}$ and mandibular movement pattern and amplitude from sensors positioned on the patients' chin and forehead. The patients received a target-controlled infusion of propofol until a "sleep" state was reached as determined by a bispectral index value of 60. Fifty four episodes of airway obstruction, defined as a $10 \mathrm{~s}$ loss of end-tidal partial pressure of $\mathrm{CO}_{2}\left(\mathrm{PETCO}_{2}\right)$ or positive RESDA signal, occurred in these patients with the proposed optimal RESDA algorithm threshold detecting OSA on average $29 \mathrm{~s}$ before end-tidal $\mathrm{CO}_{2}$ alone. Combining capnography with the mandibular movement assessment of respiratory effort shows promise to improve patient safety by providing an earlier notification of airway obstruction during propofol sedation.

Shu et al. [34] reported on 4 surgical cases that illustrate the utility of using changes in the shape of the capnogram to rapidly assess pulmonary perfusion in patients undergoing a lobectomy while receiving one lung ventilatory support. In all of these cases the appearance of a "chair-like" capnogram alerted the anesthesiologist or surgeon to a problem on the affected side and allowed for the rapid diagnosis and correction of an obstructed pulmonary artery due to a stenosed portion, a mistaken occlusion, or thrombus in the pulmonary artery or a stenosis of the anastomosis. Expanding upon the authors' conclusion, it is important to be aware of changes in the shape of the capnogram and the derived parameters (e.g. end-tidal $\mathrm{CO}_{2}$ ) and interpret those changes in the proper clinical context.

Sigmundsson et al. [35] evaluated the performance of a differential Fick method for effective pulmonary blood flow (EPBF) in pigs subjected to ischemia-reperfusion and prolonged hypercapnia from the introduction of dead space. This method, termed the "capnodynamic" method, employs ventilator derived expiratory holds as the perturbation. Since its introduction nearly 40 years ago [36], the differential Fick method and its research and commercial variants (e.g. by NICO, Philips, Wallingford, and CT) have been evaluated in different clinical environments and patient conditions. As the capnodynamic method assumes the mixed venous carbon dioxide concentration $\left(\mathrm{CmvCO}_{2}\right)$ remains constant during the measurement cycle, therapies and clinical interventions that might impact $\mathrm{CmvCO}_{2}$, as during surgery, were examined. The study found that reperfusion resulted in large changes in $\mathrm{PmvCO}_{2}$, hemodynamics, and lactate with the 
EPBF transiently increasing to three times (on average) the baseline value before returning to the baseline within $5 \mathrm{~min}$. With prolonged hypercapnia, it was reported that EPBF maintains good agreement and trending with the reference cardiac output measurement (ultrasonic flow probe). $\mathrm{EPBF}$, as a measure of the non-shunted blood flow through the lung, may be estimated by the application of the carbon dioxide Fick principle in a differential form using different perturbations, assumptions, algorithms and software-based correction factors [37, 38].

Peyton et al. [39] reported on the testing of their "redeveloped" differential carbon dioxide Fick EPBF system for breath to breath monitoring using short oscillating changes in ventilator rate to calculate periodic baseline "calibrations" at 5 to $10 \mathrm{~min}$ intervals and measurements of carbon dioxide elimination to provide estimates of cardiac output (CO) in between calibration periods. The short oscillating changes included changing the respiratory rate above and below the nominal rate over a 2 -min period while adjusting the I:E ratio seeking to maintain inspiratory duration, pressure levels and mixed venous $\mathrm{CO}_{2}$ content. They evaluated the baseline calibration method against cardiac output from a continuous thermodilution Swan-Ganz catheter (CCO Combo, Edwards Lifesciences, Irvine, CA) in 50 patients undergoing cardiac surgery or liver transplant and reported accuracy and precision that compared "favorably" to other $\mathrm{CO}$ techniques. The non-shunt EPBF was corrected to $\mathrm{CO}$ using a non-invasive pulse oximeter-based estimate of shunt fraction and the breath-by-breath measurements of cardiac output computed using the ratio of the current and previous breath carbon dioxide elimination values squared. As with this method, the capnodynamic method and any method of cardiac output estimation, it is important that the reader and user of these systems be aware of the assumptions, limitations and correction factors used [40].

\section{Lung imaging}

In 2018-2019, the journal published six papers on lung imaging covering uses of electrical impedance tomography, computed tomography and lung ultrasound including applications for measurement of extravascular lung water.

Heines and coauthors [41] have studied the use of electrical impedance tomography (EIT) for defining the Positive end-expiratory pressure (PEEP) that should be applied to patients having the Acute Respiratory Distress Syndrome. The values obtained by EIT were compared to the ones derived by the PEEP/FiO ${ }_{2}$ table used by the ARDS network and with the PEEP values proposed by the attending clinicians. EIT measurements were performed simultaneously to a PEEP step ramp, first ascending and then descending. As a cut-off the author decided that a difference of $4 \mathrm{~cm} \mathrm{H}_{2} \mathrm{O}$ in suggested PEEP should have been considered clinically relevant. The result of the study was that in $26 \%$ of cases the difference between EIT-set PEEP and the one decided by the clinician was clinically relevant and only in the $36 \%$ of cases they were equal. This conclusion keeps open the debate on the use of PEEP during ARDS: while it is clear that its application is beneficial during ARDS, its magnitude and the method to define it require further studies. EIT can be useful in understanding the dynamics of lung inflation and response of the lung to PEEP application.

The introduction of EIT in pediatric ARDS (pARDS) is discussed by Dmytrowich et al. [42] who wrote a case report dealing with PEEP optimization in a 11 years old patient. The cause of ARDS was influenza A pneumonia and her status was characterized by hypoxemia, acidosis and necessity of mechanical ventilation. The management of the case was facilitated by the application of EIT for deciding the best PEEP to be applied. The authors executed a PEEP step incremental-decremental trial. The procedure allowed to identify the entity of recruitment with PEEP application and the range of PEEP potentially associated to derecruitment. The non-invasiveness and the user-friendly sequence of operations allowed the authors to use EIT on an everyday base. This approach gave the opportunity of titrating the PEEP according to the daily status of the lung, allowing to overcome also acute episodes of desaturation determined by changes in pulmonary function.

The continuous increase in the bedside use of EIT, raised questions about the improvement of the technology and the necessity of assessing its performances in clinical scenarios. For this reason, Schullcke et al. [43] performed a simulation study in order to evaluate the severity of lung obstruction by using EIT. The simulation was conducted via software by implementing a three-dimensional model of the lung. This last was perturbated by introducing ten different degree of obstruction. One of the main results of the study was that two of the more used parameters derived from EIT analysis, Coefficient of Variation and the Global Inhomogeneity Index, were not sensitive to low levels of obstruction. Their performance was better when higher level of obstruction were simulated. The most important message conveyed by this paper is that in future research for improving EIT technology, an important role must be played by simulation studies performed on three-dimensional models of the chest.

A further example of research addressing the necessity of extracting functional information from imaging is represented by the paper by Saugel et al. [44], who studied the possibility of estimating extravascular lung water index (EVLWI) by using computed tomography. A widely used method for assessing EVLWI and other fluid status variables is based on the use of transpulmonary thermodilution (TPTD). The aim of the authors was to compare the performance of CT and TPTD in assessing these variables in an 
unselected group of 21 intensive care patients. The result was that CT-derived variables could not predict elevated TPTD-derived variables. This study, although proposes different results from other reports, has the merit of including a heterogeneous group of patients, differing also for the type of ventilatory support. The recommendation from the authors was that in this specific problem, further studies are needed for defining the role of CT as substitute of TPTD.

The different methods for assessing the lung water content were reviewed by Michard [45] in a commentary to the paper by Saugel et al. The use of CT for computing lung water presents the drawbacks bound to the exposure to radiation and the necessity of transportation to the radiology department. Methods based on TPTD should be tested in a wider population of intensive care patients and not only in selected sub-populations. However, TPTD methods have the advantage to be bedside and operator-independent technologies. Lung ultrasound methods are reaching an increasing importance in this context also because of the wide availability of training programs. New technologies based on the possibility of computing thorax impedance are currently under development and in the future they will play an important role in lung water assessment.

The application of lung ultrasound (LUS) as routine method for assessing lung morphology at the bedside is a field in rapid development. Le Goff et al. [46] compared the "classical" two-dimensional lung ultrasound (2-D LUS) with the more modern three-dimensional ultrasound (3-D LUS). The study was performed on 16 intensive care patients while a single trained operator recorded the LUS images. These last were then assessed separately by experts whose task was to classify the images as deriving from normal lungs, consolidation, pleural effusion and interstitial syndrome. The comparison revealed that 3-D LUS and 2-D LUS brought to concordant diagnoses with the partial exception of lung consolidation.

\section{Technologies and strategies for ventilation management}

In 2018-2019, the journal published four papers on technologies and strategies for ventilation management covering technologies for closed-loop control system for mechanical ventilation and prediction of postoperative pulmonary complications, and investigations of impact of obesity on pulmonary complications during robotic surgery and use of continuous positive airway pressure to limit postsurgical apnea.

Schwaiberger et al. [47] implemented and evaluated a closed-loop control system for mechanical ventilation, with the system implementing the open lung concept for performing lung recruitment and setting PEEP. Evaluation was performed in an animal model using pigs, with lung damage induced by lavage, and as such simulating the early stages of acute lung injury in lungs which can be readily recruited. The authors showed that a fully automated system could normalise the most important respiratory measurements: increasing $\mathrm{PaO}_{2} / \mathrm{FiO}_{2}$ ratio from values below $100 \mathrm{mmHg}$ to around $400 \mathrm{mmHg}$; normalising $\mathrm{pH}$ and $\mathrm{PaCO}_{2}$ levels; and reducing driving pressure from levels of $20-25 \mathrm{~cm} \mathrm{H}_{2} \mathrm{O}$ to levels between 5 and $10 \mathrm{~cm} \mathrm{H}_{2} \mathrm{O}$. The primary result of this study was to show that the approach is feasible without human intervention, and that was achieved. The authors were, however, very careful to point out that such results should not be directly extrapolated to ARDS patients who, unlike damage caused by lavage, may have lungs that are not readily recruited, and who may not be as deeply anesthetised or relaxed as the animals used in this study.

Garutti et al. [48] investigated which measurements are most predictive of postoperative pulmonary complications (PPCs) in patients' mechanically ventilated using one lung ventilation (OLV), during lung resection surgery. Following OLV, at a tidal volume of $6 \mathrm{ml} / \mathrm{kg}$ and PEEP $5 \mathrm{~cm} \mathrm{H}_{2} \mathrm{O}$, data describing clinical variables (type of anesthesia, FEV1, age, surgical duration) and cytokines were analysed in 160 patients to determine the best model for prediction of PPCs. The authors found that a model including both clinical and cytokine data was most predictive of the 37 instances of PPCs seen in these data, with respiratory infection the most common complication. An improvement in classification of $28 \%$ was seen when using both clinical and cytokine data, over each of these two data sets alone, confirming that a combination of these data provides the most accurate prediction of PPCs.

Blecha et al. [49] investigated the changes in pulmonary mechanics and oxygenation during mechanical ventilation for robotic-assisted laparoscopic prostatectomy. Patients were placed in a steep $45^{\circ}$ Trendelenburg position with intraperitoneal $\mathrm{CO}_{2}$ insufflation, with the purpose of this study to investigate the effects of obesity and these manoeuvres on pulmonary mechanics and oxygenation. All respiratory parameters were affected negatively by surgical positioning (peak pressure, driving pressure, respiratory system compliance and $\mathrm{PaO}_{2} / \mathrm{FiO}_{2}$ ratio). In addition, the detrimental effects were significantly greater in patients with BMI $>30 \mathrm{~kg} / \mathrm{m}^{2}$ with driving pressure an average of $5.3 \mathrm{~cm}$ $\mathrm{H}_{2} \mathrm{O}$ greater in patients with $\mathrm{BMI}>30 \mathrm{~kg} / \mathrm{m}^{2}$ and with values reaching almost $30 \mathrm{~cm} \mathrm{H}_{2} \mathrm{O}$ in this group. These extremely high values of driving pressure in obese patients undergoing this surgical intervention are interesting and important, but should perhaps be interpreted with some caution. For patients with high pulmonary pressures due to increases in abdominal pressure, it is likely that transpulmonary pressure represents a more accurate measurement of the potential damage to the lungs. It has recently been shown that for 
surgical procedures such as those reported by Blecha et al., that only $50 \%$ of the increased driving pressure is seen by the lungs as increased transpulmonary pressure [50], supporting the case for use of esophageal pressure measurements to monitor pulmonary mechanics in such patients.

Lim et al. [51] investigated the use of continuous positive airway pressure (CPAP) to limit apnea in patients at high risk of apnoeic episodes following surgery for transurethral bladder or prostate resection. Using a STOP-Bang questionnaire to identify patients at risk of post-operative apnea, and a commercial index quantifying the degree of apnea and hypopnea, they assigned 20 patients to post-operative treatment using CPAP and supplementary oxygen, and a further 20 to supplementary oxygen alone. The application of an average CPAP pressure of $11.4 \mathrm{~cm} \mathrm{H}_{2} \mathrm{O}$ (range 5-15 cm $\mathrm{H}_{2} \mathrm{O}$ ) resulted in a significantly lower index describing apnea and hypopnea, despite no apparent differences between the groups and no negative haemodynamic effects of CPAP. These results suggest a positive role for both CPAP, and the monitoring of apnea-hypopnea events, in the post-operative period.

\section{References}

1. Karbing DS, Perchiazzi G, Rees SE, Jaffe MB. Journal of Clinical Monitoring and Computing 2017 end of year summary: respiration. J Clin Monit Comput. 2018;32(2):197-205. https://doi. org/10.1007/s10877-018-0121-8.

2. Efrati S, Deutsch I, Gurman GM. Endotracheal tube cuff-small important part of a big issue. J Clin Monit Comput. 2012;26:5360. https://doi.org/10.1007/s10877-011-9333-x.

3. Jaillette E, Martin-Loeches I, Artigas A, Nseir S. Optimal care and design of the tracheal cuff in the critically ill patient. Ann Intensive Care. 2014;4:7. https://doi.org/10.1186/2110-5820-4-7.

4. Pisano A, Verniero L, Galdieri N, Corcione A. Assessing the correct inflation of the endotracheal tube cuff: a larger pilot balloon increases the sensitivity of the 'finger-pressure' technique, but it remains poorly reliable in clinical practice. J Clin Monit Comput. 2019;33(2):301-5. https://doi.org/10.1007/s10877-018-0158-8.

5. Özcan ATD, Döğer C, But A, Kutlu I, Aksoy ŞM. Comparison of endotracheal tube cuff pressure values before and after training seminar. J Clin Monit Comput. 2018;32(3):527-31. https://doi. org/10.1007/s 10877-017-0046-7.

6. Raft J, Lamotte AS, Maury B, Clerc-Urmès I, Baumann C, Richebé P, Bouaziz H. Reliability of cuff pressure gauges. J Clin Monit Comput. 2019;33(3):431-5. https://doi.org/10.1007/s1087 7-018-0188-2.

7. Faßbender P, Haddad A, Bürgener S, Peters J. Validation of photoplethysmography device for detection of obstructive sleep apnea in the perioperative setting. J Clin Monit Comput. 2019;33(2):341-5. https://doi.org/10.1007/s10877-018-0151-2.

8. Allen J. Photoplethysmography and its application in clinical physiological measurement. Physiol Meas. 2007;28:R1-R39.

9. Mendonça F, Mostafa SS, Ravelo-García AG, Morgado-Dias F, Penzel T. Devices for home detection of obstructive sleep apnea: a review. Sleep Med Rev. 2018;41:149-60. https://doi. org/10.1016/j.smrv.2018.02.004.

10. Kapur VK, Auckley DH, Chowdhuri S, Kuhlmann DC, Mehra R, Ramar K, Harrod CG. Clinical practice guideline for diagnostic testing for adult obstructive sleep apnea: an American Academy of Sleep Medicine Clinical practice guideline. J Clin Sleep Med. 2017;13(3):479-504. https://doi.org/10.5664/jcsm.6506.

11. Kim J, Kwon JH, Kim E, Yoo SK, Shin CS. Respiratory measurement using infrared thermography and respiratory volume monitor during sedation in patients undergoing endoscopic urologic procedures under spinal anesthesia. J Clin Monit Comput. 2019;33(4):647-56. https://doi.org/10.1007/s10877-018-0214-4.

12. Harford M, Catherall J, Gerry S, Young JD, Watkinson P. Availability and performance of image-based, non-contact methods of monitoring heart rate, blood pressure, respiratory rate, and oxygen saturation: a systematic review. Physiol Meas. 2019;40(6):06TR01. https://doi.org/10.1088/1361-6579/ab1f1d.

13. Al-Khalidi FQ, Saatchi R, Burke D, Elphick H, Tan S. Respiration rate monitoring methods: a review. Pediatr Pulmonol. 2011;46(6):523-9. https://doi.org/10.1002/ppul.21416.

14. Liu J, Ai C, Zhang B, Wang Y, Brewer LM, Ting CK, Huang D, $\mathrm{Yu}$ L. Tracheal sounds accurately detect apnea in patients recovering from anesthesia. J Clin Monit Comput. 2019;33(3):437-44. https://doi.org/10.1007/s10877-018-0192-6.

15. Penzel T, Sabil A. The use of tracheal sounds for the diagnosis of sleep apnoea. Breathe. 2017;13(2):e37-e45. https://doi. org/10.1183/20734735.008817.

16. Cavalcante AN, Martin YN, Sprung J, Imsirovic J, Weingarten TN. Low minute ventilation episodes during anesthesia recovery following intraperitoneal surgery as detected by a noninvasive respiratory volume monitor. J Clin Monit Comput. 2018;32(5):929-35. https://doi.org/10.1007/s10877-017-0093-0.

17. Schumann R, Harvey B, Zahedi F, Bonney I. Minute ventilation assessment in the PACU is useful to predict postoperative respiratory depression following discharge to the floor: a prospective cohort study. J Clin Anesth. 2019;52:93-8. https://doi. org/10.1016/j.jclinane.2018.09.005.

18. Oostveen E, MacLeod D, Lorino H, Farré R, Hantos Z, Desager K, Marchal F, ERS Task Force on Respiratory Impedance Measurements. The forced oscillation technique in clinical practice: methodology, recommendations and future developments. Eur Respir J. 2003;22(6):1026-41.

19. Zimmermann SC, Watts JC, Bertolin A, Jetmalani K, King GG, Thamrin C. Discrepancy between in vivo and in vitro comparisons of forced oscillation devices. J Clin Monit Comput. 2018;32(3):509-12. https://doi.org/10.1007/s10877-017-0050-y.

20. Becher T, Schädler D, Rostalski P, Zick G, Frerichs I, Weiler N. Determination of respiratory system compliance during pressure support ventilation by small variations of pressure support. J Clin Monit Comput. 2018;32(4):741-51. https://doi.org/10.1007/s1087 7-017-0063-6.

21. Ranieri VM, Zhang H, Mascia L, Aubin M, Lin CY, Mullen JB, Grasso S, Binnie M, Volgyesi GA, Eng P, Slutsky AS. Pressuretime curve predicts minimally injurious ventilatory strategy in an isolated rat lung model. Anesthesiology. 2000;93:1320-8.

22. Wongsurakiat $P$, Yuangtrakul N. Performance and applications of bedside visual inspection of airway pressure-time curve profiles for estimating stress index in patients with acute respiratory distress syndrome. J Clin Monit Comput. 2019;33(2):281-90. https ://doi.org/10.1007/s10877-018-0153-0.

23. Edmark L, Kostova-Aherdan K, Enlund M, Hedenstierna G. Optimal oxygen concentration during induction of general anesthesia. Anesthesiology. 2003;98:28-33.

24. Burk KM, Kuck K, Orr JA. Evaluation and application of a method for estimating nasal end-tidal $\mathrm{O}_{2}$ fraction while administering supplemental $\mathrm{O}_{2}$. J Clin Monit Comput. 2019;33:1071-80. https://doi.org/10.1007/s10877-019-00264-5.

25. Hopkins SR, Wagner PD. The multiple inert gas elimination technique (MIGET): methods in physiology. New York: Springer; 2017. https://doi.org/10.1007/978-1-4939-7441-2_1 
26. Kretzschmar M, Schilling T, Vogt A, Rothen HU, Borges JB, Hachenberg T, Larsson A, Baumgardner JE, Hedenstierna G. Multiple inert gas elimination technique by micropore membrane inlet mass spectrometry - a comparison with reference gas chromatography. J Appl Physiol. 2013;115(8):1107-18. https://doi.org/10.1152/ japplphysiol.00072.2013.

27. Gerber D, Vasireddy R, Varadarajan B, Hartwich V, Schär MY, Eberle B, Vogt A. Near-real-time pulmonary shunt and dead space measurement with micropore membrane inlet mass spectrometry in pigs with induced pulmonary embolism or acute lung failure. J Clin Monit Comput. 2019;33(6):1033-41. https://doi.org/10.1007/s1087 7-018-00245-0.

28. Lumholdt M, Damgaard KA, Christensen EF, Leutscher PDC. Mathematical arterialization of peripheral venous blood gas for obtainment of arterial blood gas values: a methodological validation study in the clinical setting. J Clin Monit Comput. 2019;33(4):733-40. https://doi.org/10.1007/s10877-018-0197-1.

29. Lumholdt M, Damgaard KA, Christensen EF, Leutscher PDC. Correction to: Mathematical arterialisation of peripheral venous blood gas for obtainment of arterial blood gas values: a methodological validation study in the clinical setting. J Clin Monit Comput. 2019. https://doi.org/10.1007/s10877-019-00314-y.

30. Rees SE, Toftegaard M, Andreassen S. A method for calculation of arterial acid-base and blood gas status from measurements in the peripheral venous blood. Comput Methods Programs Biomed. 2006;81:18-25. https://doi.org/10.1016/j.teln.2006.04.001.

31. Teng WN, Ting CK, Wang YT, Hou MC, Tsou MY, Chiang H, Lin CL. Oral capnography is more effective than nasal capnography during sedative upper gastrointestinal endoscopy. J Clin Monit Comput. 2018;32(2):321-6. https://doi.org/10.1007/s10877-017-0029-8.

32. Chang KC, Orr J, Hsu WC, Yu L, Tsou MY, Westenskow DR, Ting $\mathrm{CK}$. Accuracy of $\mathrm{CO}_{2}$ monitoring via nasal cannulas and oral bite blocks during sedation for esophagogastroduodenoscopy. J Clin Monit Comput. 2016;30(2):169-73. https://doi.org/10.1007/s1087 7-015-9696-5.

33. Ponthieu N, Coeckelenbergh S, Engelman E, Tuna T, Van Obbergh L, Barvais L. Synchronized mandibular movement and capnography: a novel approach to obstructive airway detection during procedural sedation-a post hoc analysis of a prospective study. J Clin Monit Comput. 2019;33(6):1065-70. https://doi.org/10.1007/s1087 7-018-00250-3

34. Shu J, Zhang K, Chen Y, Zhang H, Zou J, Tang Y, Dong J, Du Y. Using the capnogram to assess pulmonary perfusion during a lobectomy: case studies. J Clin Monit Comput. 2019;33(6):1061-4. https ://doi.org/10.1007/s10877-018-00249-w.

35. Sigmundsson TS, Öhman T, Hallbäck M, Redondo E, Sipmann FS, Wallin M, Oldner A, Hällsjö Sander C, Björne H. Performance of a capnodynamic method estimating effective pulmonary blood flow during transient and sustained hypercapnia. J Clin Monit Comput. 2018;32(2):311-9. https://doi.org/10.1007/s10877-017-0021-3.

36. Gedeon A, Forslund L, Hedenstierna G, Romano E. A new method for noninvasive bedside determination of pulmonary blood flow. Med Biol Eng Comput. 1980;18(4):411-8. https://doi.org/10.1007/ bf02443310.

37. Peyton PJ. Pulmonary carbon dioxide elimination for cardiac output monitoring in peri-operative and critical care patients: history and current status. J Healthc Eng. 2013;4(2):203-22. https://doi. org/10.1260/2040-2295.4.2.203.

38. Joosten A, Desebbe O, Suehiro K, Murphy LS, Essiet M, Alexander B, Fischer MO, Barvais L, Van Obbergh L, Maucort-Boulch D, Cannesson M. Accuracy and precision of non-invasive cardiac output monitoring devices in perioperative medicine: a systematic review and meta-analysis. Br J Anaesth. 2017;118(3):298-310. https://doi. org/10.1093/bja/aew461.

39. Peyton PJ, Kozub M. Performance of a second generation pulmonary capnotracking system for continuous monitoring of cardiac output. J
Clin Monit Comput. 2018;32(6):1057-64. https://doi.org/10.1007/ s10877-018-0110-y.

40. Funk DJ, Moretti EW, Gan TJ. Minimally invasive cardiac output monitoring in the perioperative setting. Anesth Analg. 2009;108(3):887-97. https://doi.org/10.1213/ane.0b013e31818ffd9 9

41. Heines SJH, Strauch U, van de Poll MCG, Roekaerts PMHJ, Bergmans DCJJ. Clinical implementation of electric impedance tomography in the treatment of ARDS: a single centre experience. J Clin Monit Comput. 2019;33(2):291-300. https://doi.org/10.1007/s1087 7-018-0164-x.

42. Dmytrowich J, Holt T, Schmid K, Hansen G. Mechanical ventilation guided by electrical impedance tomography in pediatric acute respiratory distress syndrome. J Clin Monit Comput. 2018;32(3):503-7. https://doi.org/10.1007/s10877-017-0048-5.

43. Schullcke B, Krueger-Ziolek S, Gong B, Jörres RA, Mueller-Lisse $\mathrm{U}$, Moeller K. Ventilation inhomogeneity in obstructive lung diseases measured by electrical impedance tomography: a simulation study. J Clin Monit Comput. 2018;32(4):753-61. https://doi. org/10.1007/s10877-017-0069-0.

44. Saugel B, Wildgruber M, Staudt A, Dieckmeyer M, Holzapfel K, Kaissis G, Kirov MY, Kuzkov VV, Schmid RM, Huber W. Quantitative computed tomography in comparison with transpulmonary thermodilution for the estimation of pulmonary fluid status: a clinical study in critically ill patients. J Clin Monit Comput. 2019;33(1):512. https://doi.org/10.1007/s10877-018-0144-1.

45. Michard F. Lung water assessment: from gravimetry to wearables. J Clin Monit Comput. 2019;33(1):1-4. https://doi.org/10.1007/s1087 7-018-0154-z.

46. Le Goff JM, Roger C, Louart B, Claret PG, Daurat A, Bulyez S, Lefrant JY, Bobbia X, Muller L. Lung real time three-dimensional imaging in critically ill ventilated patients: a global diagnosis concordance study. J Clin Monit Comput. 2018;32(3):513-8. https:// doi.org/10.1007/s10877-017-0042-y.

47. Schwaiberger D, Pickerodt PA, Pomprapa A, Tjarks O, Kork F, Boemke W, Francis RCE, Leonhardt S, Lachmann B. Closed-loop mechanical ventilation for lung injury: a novel physiological-feedback mode following the principles of the open lung concept. J Clin Monit Comput. 2018;32(3):493-502. https://doi.org/10.1007/s1087 7-017-0040-0.

48. Garutti I, De la Gala F, Piñeiro P, Rancan L, Vara E, Reyes A, Puente-Maestu L, Bellón JM, Simón C. Usefulness of combining clinical and biochemical parameters for prediction of postoperative pulmonary complications after lung resection surgery. J Clin Monit Comput. 2019;33(6):1043-54. https://doi.org/10.1007/s10877-01900257-4.

49. Blecha S, Harth M, Zeman F, Seyfried T, Lubnow M, Burger M, Denzinger S, Pawlik MT. The impact of obesity on pulmonary deterioration in patients undergoing robotic-assisted laparoscopic prostatectomy. J Clin Monit Comput. 2019;33(1):133-43. https:// doi.org/10.1007/s10877-018-0142-3.

50. Brandão JC, Lessa MA, Motta-Ribeiro G, Hashimoto S, Paula LF, Torsani V, Le L, Bao X, Eikermann M, Dahl DM, Deng H, Tabatabaei S, Amato MBP, Vidal-Melo MF. Global and regional respiratory mechanics during robotic-assisted laparoscopic surgery: a randomized study. Anesth Analg. 2019;129(6):1564-73. https://doi. org/10.1213/ANE.0000000000004289.

51. Lim H, Oh M, Chung YH, Ki H, Lee JJ. Effects of continuous positive airway pressure in patients at high risk of obstructive sleep apnea during propofol sedation after spinal anesthesia. J Clin Monit Comput. 2019;33(4):657-63. https://doi.org/10.1007/s1087 7-018-0202-8.

Publisher's Note Springer Nature remains neutral with regard to jurisdictional claims in published maps and institutional affiliations. 